\title{
Existence results for fractional differential inclusions with three-point fractional integral boundary conditions
}

\section{$\mathrm{XiFu}$}

"Correspondence:

fuxi1984@hotmail.com Department of Mathematics,

Shaoxing University, Shaoxing,

Zhejiang 312000, P.R. China

\begin{abstract}
This paper is concerned with fractional differential inclusions with three-point fractional integral boundary conditions. We consider the fractional differential inclusions under both convexity and nonconvexity conditions on the multivalued term. Some new existence results are obtained by using standard fixed point theorems. Two examples are given to illustrate the main results.

MSC: 34A60; 26A33; 34B15

Keywords: fractional differential inclusions; boundary value problems; existence results; multivalued maps
\end{abstract}

\section{Introduction}

Fractional differential equations have recently gained much importance and attention due to the fact that they have been proved to be valuable tools in the modeling of many physical phenomena [1-3]. For some recent developments on the existence results of fractional differential equations, we can refer, for instance, to [4-17] and the references therein.

Differential inclusions arise in the mathematical modeling of certain problems in economics, optimal control, etc. and are widely studied by many authors, see [18, 19] and the references therein. For some recent works on differential inclusions of fractional order, we refer the reader to the references $[4,5,20-29]$.

Motivated by the above papers, in this article, we study a new class of fractional boundary value problems, i.e., the following fractional differential inclusions with three-point fractional integral boundary conditions:

$$
\left\{\begin{array}{l}
{ }^{c} D^{\alpha} x(t) \in F\left(t, x(t),{ }^{c} D^{\beta} x(t)\right), \quad t \in[0,1], 1<\alpha \leq 2,0<\beta<1, \\
x(0)=0, \quad a I^{\gamma} x(\eta)+b x(1)=c, \quad 0<\eta<1,
\end{array}\right.
$$

where ${ }^{c} D^{p}$ denotes the Caputo fractional derivative of order $p, I^{q}$ the Riemann-Liouville fractional integral of order $q, F:[0,1] \times \mathbb{R} \rightarrow 2^{\mathbb{R}}$ is a multifunction and $a, b, c$ are real constants with $a \eta^{1+\gamma} \neq-b \Gamma(\gamma+2)$.

We remark that when $b=-1, c=0$ and third variable of the function $F$ in (1) vanishes, problem (1) reduces to a three-point fractional integral boundary value problem (see [17] with $F=f$ a given continuous function).

\section{囪 Springer}

O2013 Fu; licensee Springer. This is an Open Access article distributed under the terms of the Creative Commons Attribution License (http://creativecommons.org/licenses/by/2.0), which permits unrestricted use, distribution, and reproduction in any medium, provided the original work is properly cited. 
The rest of this paper is organized as follows. In Section 2 we present the notations, definitions and give some preliminary results that we need in the sequel, Section 3 is dedicated to the existence results of problem (1), in the final Section 4, two examples are given to illustrate the main results.

\section{Preliminaries}

In this section, we introduce notations, definitions and preliminary facts that will be used in the remainder of this paper.

Let $(X,\|\cdot\|)$ be a normed space. We use the notations: $P(X)=\{Y \subseteq X: Y \neq \emptyset\}, P_{c l}(X)=$ $\{Y \in P(X): Y$ closed $\}, P_{b}(X)=\{Y \in P(X): Y$ bounded $\}, P_{c p}(X)=\{Y \in P(X): Y$ compact $\}$, $P_{c p, c}(X)=\{Y \in P(X): Y$ compact, convex $\}$ and so on.

Let $A, B \in P_{c l}(X)$, the Pompeiu-Hausdorff distance of $A, B$ is defined as

$$
h(A, B)=\max \left\{\sup _{a \in A} d(a, B), \sup _{b \in B} d(b, A)\right\} .
$$

A multivalued map $F: X \rightarrow P(X)$ is convex (closed) valued if $F(x)$ is convex (closed) for all $x \in X$. $F$ is said to be completely continuous if $F(B)$ is relatively compact for every $B \in P_{b}(X) . F$ is called upper semicontinuous on $X$ if, for every $x \in X$, the set $F(x)$ is a nonempty closed subset of $X$, and for every open set $O$ of $X$ containing $F(x)$, there exists an open neighborhood $U$ of $x$ such that $F(U) \subseteq O$. Equivalently, $F$ is upper semicontinuous if the set $\{x \in X: F(x) \subseteq O\}$ is open for any open set $O$ of $X$.F is called lower semicontinuous if the set $\{x \in X: F(x) \cap O \neq \emptyset\}$ is open for each open set $O$ in $X$. If a multivalued map $F$ is completely continuous with nonempty compact values, then $F$ is upper semicontinuous if and only if $F$ has a closed graph, i.e., if $x_{n} \rightarrow x_{*}$ and $y_{n} \rightarrow y_{*}$, then $y_{n} \in F\left(x_{n}\right)$ implies $y_{*} \in F\left(x_{*}\right)[30]$.

A multivalued map $F:[0,1] \rightarrow P_{c l}(X)$ is said to be measurable if, for every $x \in X$, the function $t \rightarrow d(x, F(t))=\inf \{d(x, y): y \in F(t)\}$ is a measurable function.

Definition 2.1 A multivalued map $F: X \rightarrow P_{c l}(X)$ is called

(1) $\gamma$-Lipschitz if there exists $\gamma>0$ such that

$$
h(F(x), F(y)) \leq \gamma d(x, y) \quad \text { for each } x, y \in X
$$

(2) a contraction if it is $\gamma$-Lipschitz with $\gamma<1$.

Definition 2.2 A multivalued map $F:[0,1] \times \mathbb{R} \times \mathbb{R} \rightarrow P(\mathbb{R})$ is said to be Carathéodory if:

(1) $t \rightarrow F(t, x, y)$ is measurable for each $x, y \in \mathbb{R}$;

(2) $x \rightarrow F(t, x, y)$ is upper semicontinuous for a.e. $t \in[0,1]$.

Further, a Carathéodory function $F$ is said to be $L^{1}$-Carathéodory if

(3) for each $k>0$, there exists $\varphi_{k} \in L^{k}\left([0,1], \mathbb{R}^{+}\right)$such that

$$
\|F(t, x, y)\|=\sup \{|v|: v \in F(t, x, y)\} \leq \varphi_{k}(t)
$$

for all $|x| \leq k,|y| \leq k$ and a.e. $t \in[0,1]$.

The following lemmas will be used in the sequel. 
Lemma 2.1 (see [31]) Let $X$ be a Banach space. Let $G:[0,1] \times X \rightarrow P_{c p, c}(X)$ be an $L^{1}$ Carathéodory multivalued map and $P$ be a linear continuous map from $L^{1}([0,1], X)$ to $C([0,1], X)$, then the operator

$$
P \circ S_{G}: C([0,1], X) \rightarrow P_{c p, c}(C([0,1], X)), \quad y \mapsto\left(P \circ S_{G}\right)(y)=P\left(S_{G, y}\right)
$$

is a closed graph operator in $C([0,1], X) \times C([0,1], X)$.

Here the set of selections

$$
S_{F, x}=\left\{v \in L^{1}([0,1], \mathbb{R}): v(t) \in F(t, x(t)) \text { for a.e. } t \in[0,1]\right\} .
$$

Definition 2.3 ([32]) The Riemann-Liouville fractional integral of order $q$ for a function $f$ is defined as

$$
I^{q} f(t)=\frac{1}{\Gamma(q)} \int_{0}^{t} \frac{f(s)}{(t-s)^{1-q}} d s, \quad q>0
$$

provided the integral exists.

Definition 2.4 ([32]) For at least $n$-times differentiable function $f$, the Caputo derivative of order $q$ is defined as

$$
{ }^{c} D^{q} f(t)=\frac{1}{\Gamma(n-q)} \int_{0}^{t}(t-s)^{n-q-1} f^{(n)}(s) d s, \quad n-1<q<n, n=[q]+1,
$$

where $[q]$ denotes the integer part of the real number $q$.

Lemma 2.2 ([16]) Let $\alpha>0$, then the differential equation

$$
{ }^{c} D^{\alpha} h(t)=0
$$

has solutions $h(t)=c_{0}+c_{1} t+c_{2} t^{2}+\cdots+c_{n-1} t^{n-1}$ and

$$
I^{\alpha c} D^{\alpha} h(t)=h(t)+c_{0}+c_{1} t+c_{2} t^{2}+\cdots+c_{n-1} t^{n-1},
$$

here $c_{i} \in \mathbb{R}, i=0,1,2, \ldots, n-1, n=[\alpha]+1$.

Lemma 2.3 For any $y \in C([0,1], \mathbb{R})$, the unique solution of the three-point boundary value problem

$$
\left\{\begin{array}{l}
{ }^{c} D^{\alpha} x(t)=y(t), \quad t \in[0,1], 1<\alpha \leq 2, \\
x(0)=0, \quad a I^{\gamma} x(\eta)+b x(1)=c, \quad 0<\eta<1,
\end{array}\right.
$$

is given by

$$
x(t)=\int_{0}^{t} \frac{(t-s)^{\alpha-1}}{\Gamma(\alpha)} y(s) d s+\frac{t\left(c-b \int_{0}^{1} \frac{(1-s)^{\alpha-1}}{\Gamma(\alpha)} y(s) d s\right)}{\frac{a \eta^{1+\gamma}}{\Gamma(\gamma+2)}+b}-\frac{t a \int_{0}^{\eta} \frac{(\eta-s) \alpha+\gamma-1}{\Gamma(\alpha+\gamma)} y(s) d s}{\frac{a \eta^{1+\gamma}}{\Gamma(\gamma+2)}+b} .
$$


Proof For $1<\alpha \leq 2$ and some constants $c_{0}, c_{1} \in \mathbb{R}$, the general solution of the equation ${ }^{c} D^{\alpha} x(t)=y(t)$ can be written as

$$
x(t)=I^{\alpha} y(t)+c_{0}+c_{1} t
$$

From $x(0)=0$, it follows that $c_{0}=0$. Using the integral boundary conditions of (2), we obtain

$$
\left(\frac{a \eta^{1+\gamma}}{\Gamma(\gamma+2)}+b\right) c_{1}+a I^{\alpha+\gamma} y(\eta)+b \int_{0}^{1} \frac{(1-s)^{\alpha-1}}{\Gamma(\alpha)} y(s) d s=c .
$$

Therefore, we have

$$
c_{1}=\frac{c-b \int_{0}^{1} \frac{(1-s)^{\alpha-1}}{\Gamma(\alpha)} y(s) d s-a \int_{0}^{\eta} \frac{(\eta-s)^{\alpha+\gamma-1}}{\Gamma(\alpha+\gamma)} y(s) d s}{\frac{a \eta^{1+\gamma}}{\Gamma(\gamma+2)}+b} .
$$

Substituting the values of $c_{0}, c_{1}$, we obtain the result. This completes the proof.

Let us define what we mean by a solution of problem (1).

Definition 2.5 A function $x \in A C^{1}([0,1], \mathbb{R})$ is a solution of problem (1) if it satisfies the boundary conditions in (1) and there exists a function $f \in L^{1}([0,1], \mathbb{R})$ such that $f(t) \in$ $F\left(t, x(t),{ }^{c} D^{\beta} x(t)\right)$ a.e. on $t \in[0,1]$ and

$$
x(t)=\int_{0}^{t} \frac{(t-s)^{\alpha-1}}{\Gamma(\alpha)} f(s) d s+\frac{t\left(c-b \int_{0}^{1} \frac{(1-s)^{\alpha-1}}{\Gamma(\alpha)} f(s) d s\right)}{\frac{a \eta^{1+\gamma}}{\Gamma(\gamma+2)}+b}-\frac{t a \int_{0}^{\eta} \frac{(\eta-s)^{\alpha+\gamma-1}}{\Gamma(\alpha+\gamma)} f(s) d s}{\frac{a \eta^{1+\gamma}}{\Gamma(\gamma+2)}+b} .
$$

Let $C([0,1], \mathbb{R})$ be the space of all continuous functions defined on $[0,1]$. Define the space $\mathcal{X}=\left\{x: x\right.$ and $\left.{ }^{c} D^{\beta} x \in C([0,1], \mathbb{R}), 0<\beta<1\right\}$ endowed with the norm $\|x\|=$ $\max _{t \in[0,1]}|x(t)|+\max _{t \in[0,1]}\left|{ }^{c} D^{\beta} x(t)\right|$. Obviously, $(\mathcal{X},\|\cdot\|)$ is a Banach space.

Theorem 2.1 (Nonlinear alternative of Leray-Schauder type) Let $X$ be a Banach space, $C$ be a closed convex subset of $X, U$ be an open subset of $C$ with $0 \in U$. Suppose that $F: \bar{U} \rightarrow$ $P_{c p, c}(C)$ is an upper semicontinuous compact map. Then either (1) $F$ has a fixed point in $\bar{U}$, or (2) there are $x \in \partial U$ and $\lambda \in(0,1)$ such that $x \in \lambda F(x)$.

Theorem 2.2 (Covitz and Nadler) Let $(X, d)$ be a complete metric space. If $F: X \rightarrow P_{c l}(X)$ is a contraction, then $F$ has a fixed point.

\section{Existence results}

In this section, three existence results of problem (1) are presented. The first one concerns the convex valued case, and the others are related to the nonconvex valued case.

Now let us begin with the convex valued case.

Theorem 3.1 Suppose that the following $(\mathrm{H} 1)$, (H2) and (H3) are satisfied.

(H1) $F:[0,1] \times \mathbb{R} \times \mathbb{R} \rightarrow P_{c p, c}(\mathbb{R})$ is a Carathéodory multivalued map. 
(H2) There exist $m \in L^{\infty}\left([0,1], \mathbb{R}^{+}\right)$and $\varphi:[0, \infty) \rightarrow(0, \infty)$ continuous, nondecreasing such that

$$
\|F(t, x, y)\|=\sup \{|v|: v \in F(t, x, y)\} \leq m(t)(\varphi(|x|)+\varphi(|y|))
$$

for $x, y \in \mathbb{R}, t \in[0,1]$.

(H3) There exists a constant $M>0$ such that

$$
\frac{M}{O+\|m\|(\varphi(M)+\psi(M)) Q}>1
$$

where

$$
\begin{aligned}
& \|m\|=\sup _{t \in[0,1]}|m(t)|, \quad O=\frac{|c|}{\left|\frac{a \eta^{1+\gamma}}{\Gamma(\gamma+2)}+b\right|}\left(1+\frac{1}{\Gamma(2-\beta)}\right), \\
& Q=\left(\frac{1}{\Gamma(\alpha+1)}+\frac{1}{\Gamma(\alpha-\beta+1)}+\frac{(\Gamma(2-\beta)+1)\left(\frac{|a| \eta^{\alpha+\gamma}}{\Gamma(\alpha+\gamma+1)}+\frac{|b|}{\Gamma(\alpha+1)}\right)}{\Gamma(2-\beta)\left|\frac{a \eta^{1+\gamma}}{\Gamma(\gamma+2)}+b\right|}\right) .
\end{aligned}
$$

Then boundary value problem (1) has at least one solution on $[0,1]$.

Proof Consider the multivalued operator $N: \mathcal{X} \rightarrow P(\mathcal{X})$ defined as

$$
N(x)=\left\{h \in \mathcal{X}: h=S v, v \in S_{F, x}\right\}
$$

with

$$
\begin{aligned}
& S_{F, x}=\left\{v \in L^{1}([0,1], \mathbb{R}): v(t) \in F\left(t, x(t),{ }^{c} D^{\beta} x(t)\right) \text { for a.e. } t \in[0,1]\right\} \\
& (S v)(t)=\int_{0}^{t} \frac{(t-s)^{\alpha-1}}{\Gamma(\alpha)} v(s) d s+\frac{t\left(c-b \int_{0}^{1} \frac{(1-s)^{\alpha-1}}{\Gamma(\alpha)} v(s) d s\right)}{\frac{a \eta^{1+\gamma}}{\Gamma(\gamma+2)}+b}-\frac{t a \int_{0}^{\eta} \frac{(\eta-s)^{\alpha+\gamma-1}}{\Gamma(\alpha+\gamma)} v(s) d s}{\frac{a \eta^{1+\gamma}}{\Gamma(\gamma+2)}+b} .
\end{aligned}
$$

Clearly, by Lemma 2.3, we know that the fixed points of $N$ are solutions of problem (1). From (H1) and (H2), we have, for each $x \in \mathcal{X}$, that the set $S_{F, x}$ is nonempty [31]. Next we will show that $N$ satisfies the assumptions of the nonlinear alternative of Leray-Schauder type. The proof is given in the following five steps.

Step 1: $N(x)$ is convex valued. Since $F$ is convex valued, we know that $S_{F, x}$ is convex and therefore it is obvious that for each $x \in \mathcal{X}, N(x)$ is convex.

Step 2: $N$ maps bounded sets into bounded sets in $\mathcal{X}$. Let

$$
B_{r}=\{x \in \mathcal{X}:\|x\| \leq r\}
$$

be a bounded subset of $\mathcal{X}$. We need to prove that there exists a constant $k>0$ such that for each $x \in B_{r}$, one has $\|h\| \leq k$ for each $h \in N(x)$. Let $x \in B_{r}$ and $h \in N(x)$, then there exists $v \in S_{F, x}$ such that

$$
h(t)=(S v)(t) \quad \text { for } t \in[0,1]
$$


By simple calculations, we have

$$
\begin{aligned}
|h(t)| \leq & \int_{0}^{t} \frac{(t-s)^{\alpha-1}}{\Gamma(\alpha)}|v(s)| d s+\frac{\left(|c|+|b| \int_{0}^{1} \frac{(1-s)^{\alpha-1}}{\Gamma(\alpha)}|v(s)| d s\right)}{\left|\frac{a \eta^{1+\gamma}}{\Gamma(\gamma+2)}+b\right|} \\
& +\frac{|a| \int_{0}^{\eta} \frac{(\eta-s)^{\alpha+\gamma-1}}{\Gamma(\alpha+\gamma)}|v(s)| d s}{\left|\frac{a \eta^{1+\gamma}}{\Gamma(\gamma+2)}+b\right|} \\
\leq & \frac{\|m\|(\varphi(r)+\psi(r))}{\Gamma(\alpha+1)}+\frac{|c|}{\left|\frac{a \eta^{1+\gamma}}{\Gamma(\gamma+2)}+b\right|} \\
& +\frac{\|m\|(\varphi(r)+\psi(r))\left(\frac{|a| \eta^{\alpha+\gamma}}{\Gamma(\alpha+\gamma+1)}+\frac{|b|}{\Gamma(\alpha+1)}\right)}{\left|\frac{a \eta^{1+\gamma}}{\Gamma(\gamma+2)}+b\right|} .
\end{aligned}
$$

Similarly, we can obtain

$$
\begin{aligned}
& \left|{ }^{c} D^{\beta} h(t)\right| \\
& \leq \int_{0}^{t} \frac{(t-s)^{\alpha-\beta-1}}{\Gamma(\alpha-\beta)}|v(s)| d s+\frac{\left|c_{1}\right|}{\Gamma(2-\beta)} \\
& \leq \frac{\|m\|(\varphi(r)+\psi(r))}{\Gamma(\alpha-\beta+1)}+\frac{|c|}{\Gamma(2-\beta)\left|\frac{a \eta^{1+\gamma}}{\Gamma(\gamma+2)}+b\right|} \\
& \quad+\frac{\|m\|(\varphi(r)+\psi(r))\left(\frac{|a| \eta^{\alpha+\gamma}}{\Gamma(\alpha+\gamma+1)}+\frac{|b|}{\Gamma(\alpha+1)}\right)}{\Gamma(2-\beta)\left|\frac{a \eta^{1+\gamma}}{\Gamma(\gamma+2)}+b\right|} .
\end{aligned}
$$

Therefore, we have

$$
\begin{aligned}
|h(t)| \leq & \frac{|c|}{\left|\frac{a \eta^{1+\gamma}}{\Gamma(\gamma+2)}+b\right|}\left(1+\frac{1}{\Gamma(2-\beta)}\right)+\|m\|(\varphi(r)+\psi(r)) \\
& \times\left(\frac{1}{\Gamma(\alpha+1)}+\frac{1}{\Gamma(\alpha-\beta+1)}+\frac{(\Gamma(2-\beta)+1)\left(\frac{\left.|a|\right|^{\alpha+\gamma}}{\Gamma(\alpha+\gamma+1)}+\frac{|b|}{\Gamma(\alpha+1)}\right)}{\Gamma(2-\beta)\left|\frac{a \eta^{1+\gamma}}{\Gamma(\gamma+2)}+b\right|}\right) .
\end{aligned}
$$

Hence, we obtain

$$
\|h\| \leq O+\|m\|(\varphi(r)+\psi(r)) Q=k .
$$

Step 3: $N$ maps bounded sets into equicontinuous sets in $\mathcal{X}$. Let $B_{r}$ be as in Step 2 and $0 \leq t_{1}<t_{2} \leq 1$. Then, for each $x \in B_{r}$ and $h \in N(x)$, there exists $v \in S_{F, x}$ such that $h(t)=$ $(S v)(t)$ for $t \in[0,1]$. Since

$$
\begin{aligned}
& \left|h\left(t_{2}\right)-h\left(t_{1}\right)\right| \\
& \quad \leq\left|\int_{t_{1}}^{t_{2}} \frac{\left(t_{2}-s\right)^{\alpha-1}}{\Gamma(\alpha)} v(s) d s\right|+\left|\int_{0}^{t_{1}} \frac{\left(t_{2}-s\right)^{\alpha-1}-\left(t_{1}-s\right)^{\alpha-1}}{\Gamma(\alpha)} v(s) d s\right| \\
& \quad+\frac{\left|\left(t_{2}-t_{1}\right)\left(c-b \int_{0}^{1} \frac{(1-s)^{\alpha-1}}{\Gamma(\alpha)} v(s) d s\right)\right|}{\left|\frac{a \eta^{1+\gamma}}{\Gamma(\gamma+2)}+b\right|}+\frac{\left|\left(t_{2}-t_{1}\right) a \int_{0}^{\eta} \frac{(\eta-s)^{\alpha+\gamma-1}}{\Gamma(\alpha+\gamma)} v(s) d s\right|}{\left|\frac{a \eta^{1+\gamma}}{\Gamma(\gamma+2)}+b\right|}
\end{aligned}
$$




$$
\begin{aligned}
\leq & \frac{\|m\|(\varphi(r)+\psi(r))\left(t_{2}-t_{1}\right)^{\alpha}}{\Gamma(\alpha+1)}+\frac{\|m\|(\varphi(r)+\psi(r))\left|t_{2}^{\alpha}-\left(t_{2}-t_{1}\right)^{\alpha}-t_{1}^{\alpha}\right|}{\Gamma(\alpha+1)} \\
& +\frac{|c|\left(t_{2}-t_{1}\right)}{\left|\frac{a \eta^{1+\gamma}}{\Gamma(\gamma+2)}+b\right|}+\frac{\|m\|(\varphi(r)+\psi(r))\left(t_{2}-t_{1}\right)\left(\frac{\left.|a|\right|^{\alpha+\gamma}}{\Gamma(\alpha+\gamma+1)}+\frac{|b|}{\Gamma(\alpha+1)}\right)}{\left|\frac{a \eta^{1+\gamma}}{\Gamma(\gamma+2)}+b\right|}
\end{aligned}
$$

and

$$
\begin{aligned}
& \left|{ }^{c} D^{\beta} h\left(t_{2}\right)-{ }^{c} D^{\beta} h\left(t_{1}\right)\right| \\
& \leq\left|I^{\alpha-\beta} v\left(t_{2}\right)-I^{\alpha-\beta} v\left(t_{1}\right)+\frac{c_{1} t_{2}^{1-\beta}}{\Gamma(2-\beta)}-\frac{c_{1} t_{1}^{1-\beta}}{\Gamma(2-\beta)}\right| \\
& \leq \frac{\|m\|(\varphi(r)+\psi(r))\left(t_{2}^{\alpha-\beta}-t_{1}^{\alpha-\beta}\right)}{\Gamma(\alpha-\beta+1)}+\frac{|c|\left(t_{2}^{1-\beta}-t_{1}^{1-\beta}\right)}{\Gamma(2-\beta)\left|\frac{a \eta \eta^{1+\gamma}}{\Gamma(\gamma+2)}+b\right|} \\
& \quad+\frac{\|m\|(\varphi(r)+\psi(r))\left(t_{2}^{1-\beta}-t_{1}^{1-\beta}\right)\left(\frac{|a| \eta^{\alpha+\gamma}}{\Gamma(\alpha+\gamma+1)}+\frac{|b|}{\Gamma(\alpha+1)}\right)}{\Gamma(2-\beta)\left|\frac{a \eta^{1+\gamma}}{\Gamma(\gamma+2)}+b\right|}
\end{aligned}
$$

we deduce that

$$
\left\|h\left(t_{2}\right)-h\left(t_{1}\right)\right\| \rightarrow 0 \quad \text { as } t_{2} \rightarrow t_{1}
$$

independently of $x \in B_{r}$ and $h \in N(x)$.

Step 4: $N$ has a closed graph. Let $x_{n} \rightarrow x_{*}, h_{n} \in N\left(x_{n}\right)$ and $h_{n} \rightarrow h_{*}$, we need to show that $h_{*} \in N\left(x_{*}\right)$. Since $h_{n} \in N\left(x_{n}\right)$, there exists $v_{n} \in S_{F, x_{n}}$ such that $h_{n}(t)=\left(S v_{n}\right)(t)$ for $t \in[0,1]$. We must prove that there exists $v_{*} \in S_{F, x_{*}}$ such that $h_{*}(t)=\left(S v_{*}\right)(t)$ for $t \in[0,1]$.

Now, let us consider the continuous linear operator $P: L^{1}([0,1], \mathbb{R}) \rightarrow \mathcal{X}$

$$
v \rightarrow P(v)(t)=\int_{0}^{t} \frac{(t-s)^{\alpha-1}}{\Gamma(\alpha)} v(s) d s-\frac{b t \int_{0}^{1} \frac{(1-s)^{\alpha-1}}{\Gamma(\alpha)} v(s) d s}{\frac{a \eta^{1+\gamma}}{\Gamma(\gamma+2)}+b}-\frac{a t \int_{0}^{\eta} \frac{(\eta-s)^{\alpha+\gamma-1}}{\Gamma(\alpha+\gamma)} v(s) d s}{\frac{a \eta^{1+\gamma}}{\Gamma(\gamma+2)}+b},
$$

and denote

$$
w(t)=\frac{c t}{\frac{a \eta^{1+\gamma}}{\Gamma(\gamma+2)}+b} .
$$

Clearly, we have $S v=P v+w$ and

$$
P\left(v_{n}\right)(t)=h_{n}(t)-w(t) \rightarrow h_{*}(t)-w(t) \quad \text { in } \mathcal{X}
$$

By the definition of $P$, we have

$$
h_{n}-w \in P \circ S_{F}\left(x_{n}\right)
$$

It follows from Lemma 2.1 that $P \circ S_{F}$ is a closed graph operator. Since $x_{n} \rightarrow x_{*}$, we have

$$
h_{*}(t)-w(t)=P\left(v_{*}\right)(t)
$$

for some $v_{*} \in S_{F, x_{*}}$. This implies that $h_{*} \in N\left(x_{*}\right)$. 
Step 5: A priori bounds for solutions. Let $x \in \lambda N(x)$ for some $\lambda \in(0,1)$. Then there exists $v \in S_{F, x}$ such that $x(t)=\lambda(S v)(t)$ for $t \in[0,1]$. By a similar discussion as in Step 2, we have

$$
|x(t)|+\left|{ }^{c} D^{\beta} x(t)\right| \leq O+\|m\|(\varphi(\|x\|)+\psi(\|x\|)) Q \text { for } t \in[0,1] .
$$

Thus

$$
\|x\| \leq O+\|m\|(\varphi(\|x\|)+\psi(\|x\|)) Q \quad \text { for } t \in[0,1]
$$

By the assumption of (H3), there exists $M$ such that $\|x\| \neq M$. Let us set

$$
U=\{x \in \mathcal{X}:\|x\|<M\} .
$$

As a consequence of Steps 1-4, together with the Arzela-Ascoli theorem, we can obtain that $N: \bar{U} \rightarrow P_{c p, c}(\mathcal{X})$ is an upper semicontinuous and completely continuous map. From the choice of $U$, there is no $x \in \partial U$ such that $x \in \lambda N(x)$ for some $\lambda \in(0,1)$. Hence, by Theorem 2.1, we deduce that $N$ has a fixed point $x \in \bar{U}$ which is a solution of problem (1). This is the end of the proof.

Next we study the case when $F$ is not necessarily convex valued.

Let $A$ be a subset of $[0,1] \times \mathbb{R}$. $A$ is $\Sigma \otimes \mathcal{B}_{\mathbb{R}}$ measurable if $A$ belongs to the $\sigma$-algebra generated by all sets of the form $J \times D$, where $J$ is Lebesgue measurable in $[0,1]$ and $D$ is a Borel set of $\mathbb{R}$. A subset $A$ of $L^{1}([0,1], \mathbb{R})$ is decomposable if for all $u, v \in A$ and $J \subseteq$ $[0,1]$ Lebesgue measurable, then $u \chi_{J}+v \chi_{[0,1]-J} \in A$, where $\chi$ stands for the characteristic function.

Theorem 3.2 Let $(\mathrm{H} 2)$ and $(\mathrm{H} 3)$ hold and assume:

(H4) $F:[0,1] \times \mathbb{R} \times \mathbb{R} \rightarrow P_{c p}(\mathbb{R})$ is such that: $(1)(t, x, y) \rightarrow F(t, x, y)$ is $\Sigma \otimes \mathcal{B}_{\mathbb{R}} \otimes \mathcal{B}_{\mathbb{R}}$ measurable; (2) the map $(x, y) \rightarrow F(t, x, y)$ is lower semicontinuous for a.e. $t \in[0,1]$. Then problem (1) has at least one solution on $[0,1]$.

Proof From (H2), (H4) and Lemma 4.4 of [27], the map

$$
\mathcal{F}: \mathcal{X} \rightarrow P\left(L^{1}([0,1], \mathbb{R})\right), \quad x \rightarrow \mathcal{F}(x)=S_{F, x}
$$

is lower semicontinuous and has nonempty closed and decomposable values. Then, from a selection theorem due to Bressan and Colombo [33], there exists a continuous function $f: \mathcal{X} \rightarrow L^{1}([0,1], \mathbb{R})$ such that for all $x \in \mathcal{X}, f(x)(t) \in F\left(t, x(t),{ }^{c} D^{\beta} x(t)\right)$ a.e. $t \in[0,1]$. Now consider the problem

$$
{ }^{c} D^{\alpha} x(t)=f(x)(t), \quad t \in[0,1]
$$

with the boundary conditions in (2). Note that if $x \in \mathcal{X}$ is a solution of problem (7), then $x$ is a solution to problem (1).

Problem (7) is then reformulated as a fixed point problem for the operator $\bar{N}: \mathcal{X} \rightarrow \mathcal{X}$ defined by

$$
\bar{N}(x)(t)=(S f(x))(t) .
$$


It can easily be shown that $\bar{N}$ is continuous and completely continuous and satisfies all conditions of the Leray-Schauder nonlinear alternative for single-valued maps [34]. By a discussion similar to the one in Theorem 3.1, Theorem 3.2 follows.

\section{Theorem 3.3 We assume that:}

(H5) $F:[0,1] \times \mathbb{R} \times \mathbb{R} \rightarrow P_{c p}(\mathbb{R})$ is such that: (1) the map $t \rightarrow F(t, x, y)$ is measurable for all $x, y \in \mathbb{R} ;(2)$ there exists $m \in L^{\infty}\left([0,1], \mathbb{R}^{+}\right)$such that for a.e. $t \in[0,1]$ and all $x_{1}, x_{2}, y_{1}, y_{2} \in \mathbb{R}$,

$$
h\left(F\left(t, x_{1}, y_{1}\right), F\left(t, x_{2}, y_{2}\right)\right) \leq m(t)\left(\left|x_{1}-x_{2}\right|+\left|y_{1}-y_{2}\right|\right),
$$

and

$$
\frac{\|m\|}{\Gamma(\alpha+1)}+\frac{\|m\|}{\Gamma(\alpha-\beta+1)}+\frac{\left(\frac{\|m\| b \mid}{\Gamma(\alpha+1)}+\frac{\|m\||| a \mid}{\Gamma(\alpha+\gamma+1)}\right)(1+\Gamma(2-\beta))}{\Gamma(2-\beta)\left|\frac{a \eta^{1+\gamma}}{\Gamma(\gamma+2)}+b\right|}<1
$$

then problem (1) has at least one solution on $[0,1]$.

Proof From (H5), for each $x \in \mathcal{X}$, the multivalued map $t \rightarrow F\left(t, x(t),{ }^{c} D^{\beta} x(t)\right)$ is measurable and closed valued. Hence it has measurable selection (Theorem 2.2.1 [30]) and the set $S_{F, x}$ is nonempty. Let $N$ be defined in (5). We will show that $N$ satisfies the requirements of Theorem 2.2.

Step 1: For each $x \in \mathcal{X}, N(x) \in P_{c l}(\mathcal{X})$. Let $h_{n} \in N(x)$ be such that $h_{n} \rightarrow h$ in $\mathcal{X}$. Then $h \in \mathcal{X}$ and there exists $v_{n} \in S_{F, x}$ such that

$$
h_{n}(t)=\left(S v_{n}\right)(t), \quad t \in[0,1]
$$

By (H5), the sequence $v_{n}$ is integrable bounded. Since $F$ has compact values, we may pass to a subsequence if necessary to get that $v_{n}$ converges to $v$ in $L^{1}([0,1], \mathbb{R})$. Thus $v \in S_{F, x}$ and for each $t \in[0,1]$,

$$
h_{n}(t) \rightarrow h(t)=(S v)(t) .
$$

This implies that $h \in N(x)$ and $N(x)$ is closed.

Step 2: There exists $0<\lambda<1$ such that

$$
h(N(x), N(y)) \leq \lambda\|x-y\| \quad \text { for all } x, y \in \mathcal{X} .
$$

Let $x, y \in \mathcal{X}$ and $h_{1} \in N(y)$, then there exists $v_{1} \in S_{F, y}$ such that

$$
h_{1}(t)=\left(S v_{1}\right)(t), \quad t \in[0,1] .
$$

From (H5), we know that

$$
h\left(F\left(t, x(t),{ }^{c} D^{\beta} x(t)\right), F\left(t, y(t),{ }^{c} D^{\beta} y(t)\right)\right) \leq m(t)\left(|x(t)-y(t)|+\left|{ }^{c} D^{\beta} x(t)-{ }^{c} D^{\beta} y(t)\right|\right) .
$$


Hence, for a.e. $t \in[0,1]$, there exists $u \in F\left(t, x(t),{ }^{c} D^{\beta} x(t)\right)$ such that

$$
\left|v_{1}(t)-u\right| \leq m(t)\left(|x(t)-y(t)|+\left|{ }^{c} D^{\beta} x(t)-{ }^{c} D^{\beta} y(t)\right|\right) .
$$

Consider the multivalued map $V:[0,1] \rightarrow P(\mathbb{R})$ given by

$$
V(t)=\left\{u \in \mathbb{R}:\left|v_{1}(t)-u\right| \leq m(t)\left(|x(t)-y(t)|+\left|{ }^{c} D^{\beta} x(t)-{ }^{c} D^{\beta} y(t)\right|\right)\right\} .
$$

Since $v_{1}(t), \alpha(t)=m(t)\left(|x(t)-y(t)|+\left|{ }^{c} D^{\beta} x(t)-{ }^{c} D^{\beta} y(t)\right|\right)$ are measurable, Theorem III.41 in [35] implies that $V$ is measurable. It follows from (H5) that the map $t \rightarrow F(t, x(t))$ is measurable. Hence, by (9) and Proposition 2.1.43 in [30], the multivalued map $t \rightarrow$ $V(t) \cap F\left(t, x(t),{ }^{c} D^{\beta} x(t)\right)$ with nonempty closed values is measurable. Therefore, we can find $v_{2}(t) \in F\left(t, x(t),{ }^{c} D^{\beta} x(t)\right)$ and

$$
\left|v_{1}(t)-v_{2}(t)\right| \leq m(t)\left(|x(t)-y(t)|+\left|{ }^{c} D^{\beta} x(t)-{ }^{c} D^{\beta} y(t)\right|\right) \quad \text { for a.e. } t \in[0,1] .
$$

Let $h_{2}(t)=\left(S v_{2}\right)(t)$, i.e., $h_{2} \in N(x)$. Since

$$
\begin{aligned}
& \left|h_{1}(t)-h_{2}(t)\right| \\
& \leq \int_{0}^{t} \frac{(t-s)^{\alpha-1}}{\Gamma(\alpha)}\left|v_{1}(s)-v_{2}(s)\right| d s+\frac{\left(|b| \int_{0}^{1} \frac{(1-s)^{\alpha-1}}{\Gamma(\alpha)}\left|v_{1}(s)-v_{2}(s)\right| d s\right)}{\left|\frac{a \eta^{1+\gamma}}{\Gamma(\gamma+2)}+b\right|} \\
& \quad+\frac{\left(|a| \int_{0}^{\eta} \frac{(\eta-s)^{\alpha+\gamma-1}}{\Gamma(\alpha+\gamma)}\left|v_{1}(s)-v_{2}(s)\right| d s\right)}{\left|\frac{a \eta^{1+\gamma}}{\Gamma(\gamma+2)}+b\right|} \\
& \leq\left(\frac{\|m\|}{\Gamma(\alpha+1)}+\frac{\|m\||b|}{\Gamma(\alpha+1)\left|\frac{a \eta^{1+\gamma}}{\Gamma(\gamma+2)}+b\right|}+\frac{\|m\||a|}{\Gamma(\alpha+\gamma+1)\left|\frac{a \eta^{1+\gamma}}{\Gamma(\gamma+2)}+b\right|}\right)\|x-y\|
\end{aligned}
$$

and

$$
\begin{aligned}
& \left|{ }^{c} D^{\beta} h_{1}(t)-{ }^{c} D^{\beta} h_{2}(t)\right| \\
& \leq \int_{0}^{t} \frac{(t-s)^{\alpha-\beta-1}}{\Gamma(\alpha-\beta)}\left|v_{1}(s)-v_{2}(s)\right| d s+\frac{\left(|b| \int_{0}^{1} \frac{(1-s)^{\alpha-1}}{\Gamma(\alpha)}\left|v_{1}(s)-v_{2}(s)\right| d s\right)}{\Gamma(2-\beta)\left|\frac{a \eta^{1+\gamma}}{\Gamma(\gamma+2)}+b\right|} \\
& \quad+\frac{\left(|a| \int_{0}^{\eta} \frac{(\eta-s)^{\alpha+\gamma-1}}{\Gamma(\alpha+\gamma)}\left|v_{1}(s)-v_{2}(s)\right| d s\right)}{\Gamma(2-\beta)\left|\frac{a \eta^{1+\gamma}}{\Gamma(\gamma+2)}+b\right|} \\
& \leq\left(\frac{\|m\|}{\Gamma(\alpha-\beta+1)}+\frac{\frac{\|m\| b \mid}{\Gamma(\alpha+1)}+\frac{\|m\||| a \mid}{\Gamma(\alpha+\gamma+1)}}{\Gamma(2-\beta)\left|\frac{a \eta^{1+\gamma}}{\Gamma(\gamma+2)}+b\right|}\right)\|x-y\|,
\end{aligned}
$$

we obtain that

$$
\begin{aligned}
& \left\|h_{1}(t)-h_{2}(t)\right\| \\
& \leq\left(\frac{\|m\|}{\Gamma(\alpha+1)}+\frac{\|m\|}{\Gamma(\alpha-\beta+1)}+\frac{\frac{\|m\||b|}{\Gamma(\alpha+1)}(1+\Gamma(2-\beta))}{\Gamma(2-\beta)\left|\frac{a \eta^{1+\gamma}}{\Gamma(\gamma+2)}+b\right|}\right. \\
& \left.+\frac{\frac{\|m\||a|}{\Gamma(\alpha+\gamma+1)}(1+\Gamma(2-\beta))}{\Gamma(2-\beta)\left|\frac{a \eta^{1+\gamma}}{\Gamma(\gamma+2)}+b\right|}\right)\|x-y\| .
\end{aligned}
$$


Define

$$
\lambda=\frac{\|m\|}{\Gamma(\alpha+1)}+\frac{\|m\|}{\Gamma(\alpha-\beta+1)}+\frac{\left(\frac{\|m\| b \mid}{\Gamma(\alpha+1)}+\frac{\|m\||| a \mid}{\Gamma(\alpha+\gamma+1)}\right)(1+\Gamma(2-\beta))}{\Gamma(2-\beta)\left|\frac{a \eta^{1+\gamma}}{\Gamma(\gamma+2)}+b\right|} .
$$

By using an analogous relation obtained by interchanging the roles of $x$ and $y$, we get

$$
h(N(x), N(y)) \leq \lambda\|x-y\| .
$$

Therefore from condition (8), Theorem 2.2 implies that $N$ has a fixed point which is a solution of problem (1). This completes the proof.

\section{Examples}

In this section, we give two examples to illustrate the results.

Example 1 Consider the following three-point fractional integral boundary value problem:

$$
\left\{\begin{array}{l}
{ }^{c} D^{\frac{3}{2}} x(t) \in F\left(t, x(t),{ }^{c} D^{\frac{3}{4}} x(t)\right), \quad t \in[0,1] \\
x(0)=0, \quad I^{\frac{4}{3}} x\left(\frac{1}{2}\right)+\frac{1}{3} x(1)=-1,
\end{array}\right.
$$

where $\alpha=\frac{3}{2}, \beta=\frac{3}{4}, \gamma=\frac{4}{3}, \eta=\frac{1}{2}, a=1, b=\frac{1}{3}, c=-1$ and $F:[0,1] \times \mathbb{R} \times \mathbb{R} \rightarrow P(\mathbb{R})$ is a multivalued map given by

$$
F(t, x, y)=\left\{v \in \mathbb{R}: e^{-|x|}-\frac{|y|}{1+y^{2}}+\sin t+t^{2} \leq v \leq 4+\frac{|x|}{1+x^{2}}+\sin y+6 t^{3}\right\} .
$$

In the context of this problem, we have

$$
\|F(t, x, y)\|=\sup \{|v|: v \in F(t, x, y)\} \leq 6+6 t^{2} \leq 12 \quad \text { for } t \in[0,1], x, y \in \mathbb{R} .
$$

It is clear that $\mathrm{F}$ is convex compact valued and is of Carathéodory type. Let $m(t) \equiv 1$ and $\varphi(|x|) \equiv 4, \psi(|y|) \equiv 8$, we get that for $t \in[0,1], x, y \in \mathbb{R}$,

$$
\|F(t, x, y)\|=\sup \{|v|: v \in F(t, x, y)\} \leq m(t)(\varphi(|x|)+\varphi(|y|)) .
$$

As for condition (4), since $O+\|m\|(\varphi(|x|)+\psi(|y|)) Q=O+12 Q$ (see $O, Q$ in $(\mathrm{H} 3))$ is a constant, we can choose $M$ large enough so that

$$
\frac{M}{O+\|m\|(\varphi(|M|)+\psi(|M|)) Q}>1
$$

Thus, by the conclusion of Theorem 3.1, boundary value problem (10) has at least one solution on $[0,1]$.

Example 2 Consider the following three-point fractional integral boundary value problem:

$$
\left\{\begin{array}{l}
{ }^{c} D^{\frac{7}{4}} x(t) \in F\left(t, x(t),{ }^{c} D^{\frac{1}{2}} x(t)\right), \quad t \in[0,1], \\
x(0)=0, \quad I^{\frac{4}{3}} x\left(\frac{1}{3}\right)+\frac{2}{3} x(1)=-\frac{1}{2},
\end{array}\right.
$$


where $\alpha=\frac{7}{4}, \beta=\frac{1}{2}, \gamma=\frac{4}{3}, \eta=\frac{1}{3}, a=1, b=\frac{2}{3}, c=-\frac{1}{2}$,

$$
F(t, x, y)=\left[-l_{1}(t)-\frac{\sin x}{(4+t)^{2}}-3,-\frac{1}{10}\right] \cup\left[0, \frac{|y|}{16(1+|y|)}+l_{2}(t)\right],
$$

and $l_{1}, l_{2} \in L^{1}\left([0,1], \mathbb{R}^{+}\right)$.

From the data given above, we have

$$
\begin{aligned}
& \sup \{|v|: v \in F(t, x, y)\} \leq 4+\frac{1}{(4+t)^{2}}+l_{1}(t)+l_{2}(t), \quad t \in[0,1], x, y \in \mathbb{R}, \\
& h\left(F\left(t, x_{1}, y_{1}\right), F\left(t, x_{2}, y_{2}\right)\right) \leq \frac{1}{16}\left(\left|x_{1}-x_{2}\right|+\left|y_{1}-y_{2}\right|\right) .
\end{aligned}
$$

Let $m=\frac{1}{16}$, we can get

$$
\frac{1}{16}\left(\frac{1}{\Gamma\left(\frac{11}{4}\right)}+\frac{1}{\Gamma\left(\frac{9}{4}\right)}+\frac{\frac{\frac{2}{3}}{\Gamma\left(\frac{11}{4}\right)}\left(1+\Gamma\left(\frac{3}{2}\right)\right)}{\Gamma\left(\frac{3}{2}\right)\left|\frac{\frac{1}{3}^{\frac{7}{3}}}{\Gamma\left(\frac{10}{3}\right)}+\frac{2}{3}\right|}+\frac{\frac{1}{\Gamma\left(\frac{49}{12}\right)}\left(1+\Gamma\left(\frac{3}{2}\right)\right)}{\Gamma\left(\frac{3}{2}\right)\left|\frac{\frac{1}{3}^{\frac{7}{3}}}{\Gamma\left(\frac{10}{3}\right)}+\frac{2}{3}\right|}\right) \approx 0.2841<1
$$

Hence it follows from Theorem 3.3 that problem (11) has at least one solution on $[0,1]$.

\section{Competing interests}

The author declares that he has no competing interests.

\section{Author's contributions}

The author carried out the proofs of the theorems and approved the final manuscript.

\section{Acknowledgements}

The author would like to express his thanks to the referees for their helpful suggestions.

Received: 15 May 2013 Accepted: 16 October 2013 Published: 08 Nov 2013

\section{References}

1. Băleanu, D, Machado, JAT, Luo, ACJ: Fractional Dynamics and Control. Springer, Berlin (2012)

2. Sabatier, J, Agrawal, OP, Machado, JAT (eds.): Advances in Fractional Calculus: Theoretical Developments and Applications in Physics and Engineering. Springer, Dordrecht (2007)

3. Lakshmikantham, V, Leela, S, Vasundhara Devi, J: Theory of Fractional Dynamic Systems. Cambridge Scientific Publishers, Cambridge (2009)

4. Agarwal, RP, Belmekki, M, Benchohra, M: A survey on semilinear differential equations and inclusions involving Riemann-Liouville fractional derivative. Adv. Differ. Equ. 2009, Article ID 981728 (2009)

5. Agarwal, RP, Benchohra, M, Hamani, S: A survey on existence results for boundary value problems of nonlinear fractional differential equations and inclusions. Acta Appl. Math. 109, 973-1033 (2010)

6. Bai, Z: On positive solutions of a nonlocal fractional boundary value problem. Nonlinear Anal. 72(2), 916-924 (2010)

7. Chang, Y, Nieto, JJ: Some new existence results for fractional differential inclusions with boundary conditions. Math. Comput. Model. 49, 605-609 (2009)

8. Chen, A, Tian, Y: Existence of three positive solutions to three-point boundary value problem of nonlinear fractional differential equation. Differ. Equ. Dyn. Syst. 18(3), 327-339 (2010)

9. Chen, Y, Tang, X: Positive solutions of fractional differential equations at resonance on the half-line. Bound. Value Probl. 2012, Article ID 64 (2012)

10. Li, CF, Luo, XN, Zhou, Y: Existence of positive solutions of the boundary value problem for nonlinear fractional differential equations. Comput. Math. Appl. 59, 1363-1375 (2010)

11. Ahmad, B, Nieto, JJ: Anti-periodic fractional boundary value problems. Comput. Math. Appl. 62, 1150-1156 (2011)

12. Wang, G, Ahmad, B, Zhang, L: Impulsive anti-periodic boundary value problem for nonlinear differential equations of fractional order. Nonlinear Anal. 74(3), 792-804 (2011)

13. Wang, JR, Zhou, Y: Existence and controllability results for fractional semilinear differential inclusions. Nonlinear Anal., Real World Appl. 12(6), 3642-3653 (2011)

14. Wang, JR, LV, L, Zhou, Y: Boundary value problems for fractional differential equations involving Caputo derivative in Banach spaces. J. Appl. Math. Comput. 38, 209-224 (2012)

15. Zhou, Y, Jiao, F: Nonlocal Cauchy problem for fractional evolution equations. Nonlinear Anal. 11, 4465-4475 (2010) 
16. Zhang, S: Positive solutions for boundary-value problems of nonlinear fractional differential equations. Electron. J. Differ. Equ. 36, 1-12 (2006)

17. Sudsutad, W, Tariboon, J: Boundary value problems for fractional differential equations with three-point fractional integral boundary conditions. Adv. Differ. Equ. 2012, Article ID 93 (2012)

18. Smirnov, GV: Introduction to the Theory of Differential Inclusions. Am. Math. Soc., Providence (2002)

19. Tolstonogov, AA: Differential Inclusions in a Banach Space. Kluwer Academic, Dordrecht (2000)

20. Cernea, A: A note on the existence of solutions for some boundary value problems of fractional differential inclusions. Fract. Calc. Appl. Anal. 15(2), 183-194 (2012)

21. Cernea, A: On the existence of solutions for fractional differential inclusions with anti-periodic boundary conditions. J. Appl. Math. Comput. 38, 133-143 (2012)

22. Ahmad, B: Existence results for fractional differential inclusions with separated boundary conditions. Bull. Korean Math. Soc. 47(4), 805-813 (2010)

23. Ahmad, B, Ntouyas, SK: Fractional differential inclusions with fractional separated boundary conditions. Fract. Calc. Appl. Anal. 15(3), 362-382 (2012)

24. Hamani, S, Benchohra, M, Graef, JR: Existence results for boundary-value problems with nonlinear fractional differential inclusions and integral conditions. Electron. J. Differ. Equ. 2010(20), 1-16 (2010)

25. Ahmad, B, Ntouyas, SK: Some existence results for boundary value problems of fractional differential inclusions with non-separated boundary conditions. Electron. J. Qual. Theory Differ. Equ. 2010(71), 1-17 (2010)

26. Cernea, A: Some remarks on a fractional differential inclusion with non-separated boundary conditions. Electron. J. Qual. Theory Differ. Equ. 2011(45), 1-14 (2011)

27. Ouahab, A: Some results for fractional boundary value problem of differential inclusions. Nonlinear Anal. 69, 3877-3896 (2008)

28. Nyamoradi, N, Javidi, M: Existence of multiple positive solutions for fractional differential inclusions with $m$-point boundary conditions and two fractional orders. Electron. J. Differ. Equ. 2012(187), 1-26 (2012)

29. Nyamoradi, N, Baleanu, D, Agarwal, RP: On a multipoint boundary value problem for a fractional order differential inclusion on an infinite interval. Adv. Math. Phys. 2013, Article ID 823961 (2013)

30. Hu, SC, Papageorgiou, NS: Handbook of Multivalued Analysis, Volume I: Theory. Kluwer Academic, Dordrecht (1997)

31. Lasota, A, Opial, Z: An application of the Kakutani-Ky Fan theorem in the theory of ordinary differential equations. Bull. Acad. Pol. Sci., Sér. Sci. Math. Astron. Phys. 13, 781-786 (1965)

32. Kilbas, AA, Srivastava, HM, Trujillo, JJ: Theory and Applications of Fractional Differential Equations. North-Holland Mathematics Studies, vol. 204. Elsevier, Amsterdam (2006)

33. Bressan, A, Colombo, G: Extensions and selections of maps with decomposable values. Stud. Math. 90, 69-86 (1988)

34. Granas, A, Dugundji, J: Fixed Point Theory. Springer, New York (2003)

35. Castaing, C, Valadier, M: Convex Analysis and Measurable Multifunctions. Springer, New York (1997)

10.1186/1687-1847-2013-304

Cite this article as: Fu: Existence results for fractional differential inclusions with three-point fractional integral

boundary conditions. Advances in Difference Equations 2013, 2013:304 